\title{
Infrared spectroscopy of molecular ions in selected rotational and spin-orbit states
}

\section{Journal Article}

\section{Author(s):}

Jacovella, Ugo; Agner, Josef A.; Schmutz, Hansjürg; Deiglmayr, Johannes; Merkt, Frédéric

Publication date:

2016-07

Permanent link:

https://doi.org/10.3929/ethz-b-000118387

\section{Rights / license:}

In Copyright - Non-Commercial Use Permitted

\section{Originally published in:}

The Journal of Chemical Physics 145(1), https://doi.org/10.1063/1.4954701

\section{Funding acknowledgement:}

159848 - Rydberg states, VUV laser spectroscopy and photoionization dynamics (SNF) 
This article may be downloaded for personal use only. Any other use requires prior permission of the author and AIP Publishing.

The following article appeared in J. Chem. Phys. 145, 014301 (2016) and may be found at http://dx.doi.org/10.1063/1.4954701. 


\title{
Infrared spectroscopy of molecular ions in selected rotational and spin-orbit states
}

\author{
${ }_{3}$ U. Jacovella, ${ }^{1}$ J. A. Agner, ${ }^{1}$ H. Schmutz, ${ }^{1}$ J. Deiglmayr, ${ }^{1}$ and F. Merkt ${ }^{1, a)}$ \\ 4 \\ Laboratorium für Physikalische Chemie, ETH Zürich, CH-8093 Zurich, Switzerland
}

(Dated: 19 November 2019)

First results are presented obtained with an experimental setup developed to record IR spectra of rotationally state-selected ions. The method we use is a state-selective version of a method developed by S. Schlemmer, D. Gerlich and coworkers (Int. J. Mass. Spec. 185, 589 (1999); J. Chem. Phys. 117, 2068 (2002)) to record IR spectra of ions. Ions are produced in specific rotational levels using mass-analyzed-threshold-ionization spectroscopy. The state-selected ions generated by pulsed-field ionization of Rydberg states of high principal quantum number $(n \approx 200)$ are extracted toward an octupole ion guide containing a neutral target gas. Prior to entering the octupole, the ions are excited by an IR laser. The target gas is chosen so that only excited ions react to form product ions. These product ions are detected mass selectively as a function of the IR laser wavenumber. To illustrate this method, we present IR spectra of $\mathrm{C}_{2} \mathrm{H}_{2}^{+}$in selected rotational levels of the ${ }^{2} \Pi_{\mathrm{u}, 3 / 2}$ and ${ }^{2} \Pi_{\mathrm{u}, 1 / 2}$ spin-orbit components of the vibronic ground state.

6 PACS numbers: Valid PACS appear here

$7 \quad$ Keywords: Suggested keywords

\section{I. INTRODUCTION}

9 Many important molecular cations such as $\mathrm{H}_{3}^{+}, \mathrm{CH}_{4}^{+}$, ${ }_{10} \mathrm{CH}_{5}^{+}, \mathrm{C}_{2} \mathrm{H}_{4}^{+}, \mathrm{C}_{2} \mathrm{H}_{5}^{+}, \mathrm{C}_{2} \mathrm{H}_{6}^{+}, \mathrm{C}_{5} \mathrm{H}_{5}^{+}, \mathrm{C}_{6} \mathrm{H}_{6}^{+}, \mathrm{O}_{3}^{+}$, etc., have 11 complex or unknown spectra. The presence of unpaired 12 electrons, orbital degeneracy and/or large-amplitude mo13 tions in these ions leads to intricate energy-level struc14 tures and congested spectra. Once measured, the spec15 tra can resist assignments for years, if not for decades. ${ }_{16}$ Striking examples include the Carrington-Buttenshaw${ }_{17}$ Kennedy spectrum of $\mathrm{H}_{3}^{+},{ }^{1}$ the White-Tang-Oka spec${ }_{18}$ trum of $\mathrm{CH}_{5}^{+},{ }^{2}$ and the spectrum of $\mathrm{CH}_{4}^{+} \cdot 3,4$

${ }_{19}$ The difficulties in studying cations arise from their 20 high reactivity and the fact that space-charge effects limit 21 their densities in the gas phase. In most cases, ions are 22 generated in hot environments so that the population 23 is distributed over many quantum states, which further ${ }_{24}$ reduces the sensitivity of spectroscopic experiments and ${ }_{25}$ complicates spectral assignments.

26 Several approaches have been developed to overcome ${ }_{27}$ these difficulties. These can be classified into three 28 categories: i) the direct measurement of ion spectra 29 by absorption or emission spectroscopy, ${ }^{5-8}$ the sensitiv30 ity of which is greatly enhanced by exploiting mod31 ulation techniques, ${ }^{9-11}$ ii) the indirect measurement 32 of ion spectra by photoelectron spectroscopy of the 33 neutral parent molecules, for instance by pulsed-field34 ionization zero-kinetic-energy (PFI-ZEKE) photoelec35 tron spectroscopy, ${ }^{12,13}$ and iii) the measurement of spec36 tra by detecting photoinduced processes, e.g. dissoci37 ation products, ${ }^{14,15}$ reaction products, ${ }^{16,17}$ the suppres38 sion of complex formation, ${ }^{18,19}$ or specific processes in 39 ion traps resulting from photoexcitation ${ }^{20}$. A variant

\footnotetext{
a) Electronic mail: frederic.merkt@phys.chem.ethz.ch
}

40 of iii) consists of recording the spectra of weakly bound 41 complexes of the ion of interest with rare gas atoms and 42 monitoring the rare-gas-atom loss. ${ }^{21}$ Significant advances 43 could be made in this last category with the introduction 44 of 22-pole ion traps in combination with buffer-gas cool${ }_{45}$ ing $^{22}$ and the use of sympathetically cooled molecular 46 ions in laser-cooled Coulomb crystals. ${ }^{23}$ Several recent 47 review articles provide an overview of this very active 48 field. ${ }^{24,25}$

49 We present here a method for recording spectra of 50 state-selected ions and illustrate it with the example of 51 a measurement of the asymmetric-stretch fundamental 52 band $\left(3_{0}^{1}\right)$ of $\mathrm{C}_{2} \mathrm{H}_{2}^{+}$. The transitions are detected by mon53 itoring the $\mathrm{C}_{2} \mathrm{H}_{3}^{+}$product of the reaction

$$
\mathrm{C}_{2} \mathrm{H}_{2}^{+}\left(3^{1}, \Omega^{+}, J^{+}\right)+\mathrm{H}_{2} \rightarrow \mathrm{C}_{2} \mathrm{H}_{3}^{+}+\mathrm{H}
$$

54 which is not observable for $\mathrm{C}_{2} \mathrm{H}_{2}^{+}$ions in their vibronic 55 ground state, as was already exploited in the pioneer${ }_{56}$ ing experiment of Schlemmer et al. ${ }^{17}$. In Eq. (1), $J^{+}$is 57 the total-angular-momentum quantum number exclud58 ing nuclear spin and $\Omega^{+}$is the quantum number asso59 ciated with the projection of $J^{+}$on the molecular axis. ${ }_{60} \mathrm{C}_{2} \mathrm{H}_{2}^{+}$is particularly well suited to test the performance ${ }_{61}$ of our method because the transition frequencies are ac${ }_{62}$ curately known from the work of Oka and coworkers ${ }^{26}$ 63 and the spin-orbit and rotational structure of the pho64 toelectron spectrum of the $\mathrm{X}^{+} \leftarrow \mathrm{X}$ ionizing transi65 tion has been fully resolved by PFI-ZEKE photoelectron 66 spectroscopy $^{27}$.

67 Carrying out the experiments with state-selected ions 68 makes the spectral assignments straightforward, reduces ${ }_{69}$ spectral congestion and allows in principle the measure70 ment of infrared spectra of vibrationally excited ions. 


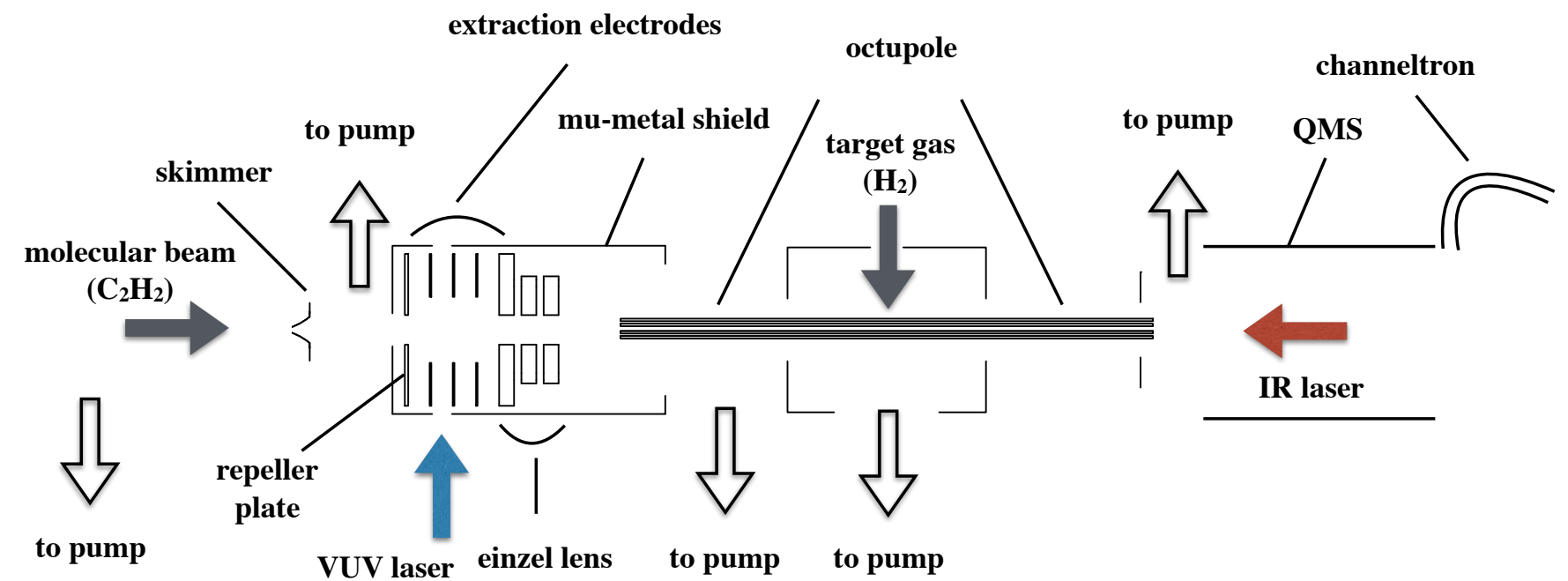

FIG. 1. Schematic diagram of the experimental setup. Different components are not to scale. See text for explanations.

\section{II. EXPERIMENTAL PROCEDURE}

72 The first step of our experimental procedure con73 sists in preparing the $\mathrm{C}_{2} \mathrm{H}_{2}^{+}$cations in selected rota74 tional levels of the ground electronic state and gener75 ating a beam of these ions. This step is accomplished 76 using mass-analyzed-threshold-ionization (MATI) ${ }^{28,29}$ 77 combined with single-photon excitation of a cold sample 78 of $\mathrm{C}_{2} \mathrm{H}_{2}$ molecules in a supersonic beam using narrow79 band vacuum-ultraviolet (VUV) laser radiation.

80 The $\mathrm{C}_{2} \mathrm{H}_{2}^{+}$ions are irradiated with an IR laser pulse ${ }_{81}$ before being extracted toward an octupole ion guide con${ }_{82}$ taining the neutral $\mathrm{H}_{2}$ target gas. The IR transitions are 83 detected by monitoring the $\mathrm{C}_{2} \mathrm{H}_{3}^{+}$product-ion yield as a ${ }_{84}$ function of the IR-laser wavenumber. The experimental 85 setup is represented schematically in Fig. 1.

86 The pulsed tunable VUV laser radiation (repeti87 tion rate $25 \mathrm{~Hz}$, pulse duration $2 \mathrm{~ns}$ ) was generated 88 by resonance-enhanced difference-frequency mixing in a 89 pulsed beam of xenon ${ }^{27}$ using the $(5 \mathrm{p})^{6} \rightarrow(5 \mathrm{p})^{5} 6 \mathrm{p}[1 / 2]_{0}$ ${ }_{90}$ two-photon resonance of Xe at $2 \tilde{\nu}_{1}=80118.98 \mathrm{~cm}^{-1}$ and 91 two commercial single-grating dye lasers and a $\beta$-barium92 borate doubling crystal.

93 The pulsed tunable IR laser radiation was generated 94 by difference-frequency mixing in a $\mathrm{KTiOAsO}_{4}$ (KTA) ${ }_{95}$ crystal. The KTA was cut at angles of $\Theta=43^{\circ}, \Phi=0^{\circ}$ 96 and had a dimension of $5 \times 5 \times 15 \mathrm{~mm}^{3}$. The seeded ${ }_{97} 532 \mathrm{~nm}$ output of the Nd:YAG laser and the output of 98 a double-grating tunable dye laser operated in the range 99 638-642 nm were overlapped using a dichroic mirror and 100 directed into the crystal. To separate the generated IR 101 laser beam from the input laser beams, these were aligned 137 102 so as to intersect in the KTA crystal at a small angle ${ }_{103}\left(<5^{\circ}\right)$. A slit was used to block the $532 \mathrm{~nm}$ and $640 \mathrm{~nm}$ 104 laser beams but transmit the IR laser beam before the IR
105 beam entered the experimental chamber through a $\mathrm{CaF}_{2}$ 106 window. The IR radiation had a measured bandwidth of $1070.035 \mathrm{~cm}^{-1}$ and a pulse energy of $200 \mu \mathrm{J}$. Its wavenumber 108 was calibrated at an absolute accuracy of $0.02 \mathrm{~cm}^{-1}$ by 109 measuring the wavenumber of the $532 \mathrm{~nm}$ and $640 \mathrm{~nm}$ 110 lasers with a wavelength meter.

A pulsed valve was employed to form a supersonic 112 beam of a mixture of $20 \%$ acetylene and $80 \%$ argon (vol113 ume \%). The beam was skimmed prior to entering the 114 photoionization chamber, where it intersected the VUV 115 laser beam at right angles on the axis of a set of five 116 parallel cylindrical extraction plates. ${ }^{27}$

The ions were state selected using two succes118 sive electric-field pulses, ${ }^{29}$ a discrimination pulse of ${ }_{119}+690 \mathrm{mV} / \mathrm{cm}$, which removed all prompt ions produced 120 by the VUV laser, followed by a field-ionization and ion121 extraction pulse of $+5.5 \mathrm{~V} / \mathrm{cm}$, generated by applying a 122 pulsed electric potential of $+32 \mathrm{~V}$ on the repeller plate ${ }_{123}$ (see Fig. 1) of the extraction region. The IR excitation 124 was carried out either during the discrimination pulse or 125 in the field-free interval between discrimination and ex126 traction pulses, or immediately following field ionization 127 with the extraction pulse. In this way the Doppler shift of 128 the IR transition only originated from the velocity of the 129 supersonic beam $(\approx 600 \mathrm{~m} / \mathrm{s})$, and was $\approx-0.006 \mathrm{~cm}^{-1}$ 130 at $3000 \mathrm{~cm}^{-1}$, i.e., more than $\approx 5$ times less than the 131 IR-laser bandwidth. The IR spectra turned out not to 132 be sensitive to the exact timing of the IR laser pulse as 133 long as the IR excitation took place before significant ac${ }_{134}$ celeration of $\mathrm{C}_{2} \mathrm{H}_{2}^{+}$by the extraction pulse. We therefore 135 conclude that the presence of the Rydberg electron has 136 no effect on IR transition frequencies within the preci37 sion limit of our experiment and of electric fields up to ${ }_{38}+5.5 \mathrm{~V} / \mathrm{cm}$. However, we observed a slight broadening 139 (from $0.035 \mathrm{~cm}^{-1}$ to $0.050 \mathrm{~cm}^{-1}$ ) of the IR transitions 140 recorded before the field-ionization pulse (see below). 
${ }_{141}$ The ions extracted toward the reaction zone were pro${ }_{142}$ duced at a potential of $\approx 18 \mathrm{~V}$, determined by the ap${ }_{143}$ plied potential and the experimental geometry, and were 144 slowed down on their way to the octupole containing the ${ }_{145}$ target gas $\mathrm{H}_{2}$ by applying $\mathrm{a} \approx 17 \mathrm{~V}$ bias potential to the ${ }_{146}$ octupole rods. The exact value of the bias potential was ${ }_{147}$ carefully adjusted so that i) the kinetic energy of the ions ${ }_{148}$ entering the reaction zone was too low for a reaction to 149 occur without prior excitation of $\mathrm{C}_{2} \mathrm{H}_{2}^{+}$with the IR laser, 150 and ii) the prompt ions were all rejected.

151 The target gas $\mathrm{H}_{2}$ was introduced from the side using 152 a pulsed valve with orifice located $10 \mathrm{~cm}$ away from the 153 octupole axis. Rather than monitoring the product ions 154 resulting from the reaction of $\mathrm{C}_{2} \mathrm{H}_{2}^{+}$with $\mathrm{H}_{2}$ molecules 155 in the supersonic beam, we found it more efficient to 156 open the $\mathrm{H}_{2}$ valve early and to let the $\mathrm{H}_{2}$ molecules ther${ }_{157}$ malize to $300 \mathrm{~K}$ prior to the arrival of the $\mathrm{C}_{2} \mathrm{H}_{2}^{+}$beam 158 in the octupole. The optimal valve-opening time was 159 determined by monitoring the $\mathrm{C}_{2} \mathrm{H}_{3}^{+}$product-ion yield, 160 as illustrated in Fig. 2. In this figure, the vertical line 161 indicates the temporal position of the VUV laser pulse 162 and the stars represent the $\mathrm{C}_{2} \mathrm{H}_{3}^{+}$ion signal. The maxi163 mum yield was obtained by opening the $\mathrm{H}_{2}$ valve about ${ }_{164} 6 \mathrm{~ms}$ before the VUV laser pulse. After the reaction, the 165 product ions $\left(\mathrm{C}_{2} \mathrm{H}_{3}^{+}\right)$were guided toward a commercial ${ }_{166}$ quadrupole mass spectrometer coupled with an off-axis ${ }_{167}$ channeltron, where they were detected mass-selectively.

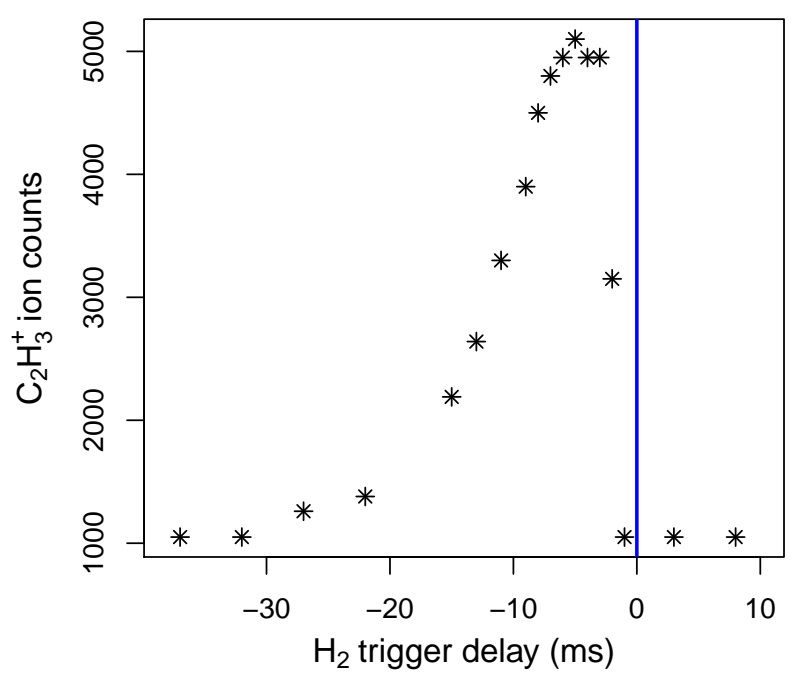

FIG. 2. $\mathrm{C}_{2} \mathrm{H}_{3}^{+}$product yield (obtained from the average of 600 experimental cycles) as a function of the $\mathrm{H}_{2}$ valve opening trigger time with respect to the VUV laser pulse (vertical line). To induce the reaction for this measurement, the $\mathrm{C}_{2} \mathrm{H}_{2}^{+}$ ions were accelerated to a kinetic energy sufficient to overcome the barrier without IR laser.

\section{RESULTS}

169 All assignments are presented using Hund's case (a) 170 nomenclature, which is adequate for low values of $\mathrm{J}^{+}$. ${ }_{171}$ The IR transitions are labeled $\mathrm{R}_{1,2}\left(J^{+}\right)$, where $\mathrm{R}$ is the 172 standard notation for transitions associated with $\Delta J^{+}$ ${ }_{173}=+1, J^{+}$is the rotational quantum number of the initial 174 state and the subscripts 1 and 2 refer to the $F_{1}\left({ }^{2} \Pi_{3 / 2}\right)$ 175 and $\mathrm{F}_{2}\left({ }^{2} \Pi_{1 / 2}\right)$ spin-orbit components of the $\mathrm{X}^{+}$ground 176 electronic state of $\mathrm{C}_{2} \mathrm{H}_{2}^{+}$.

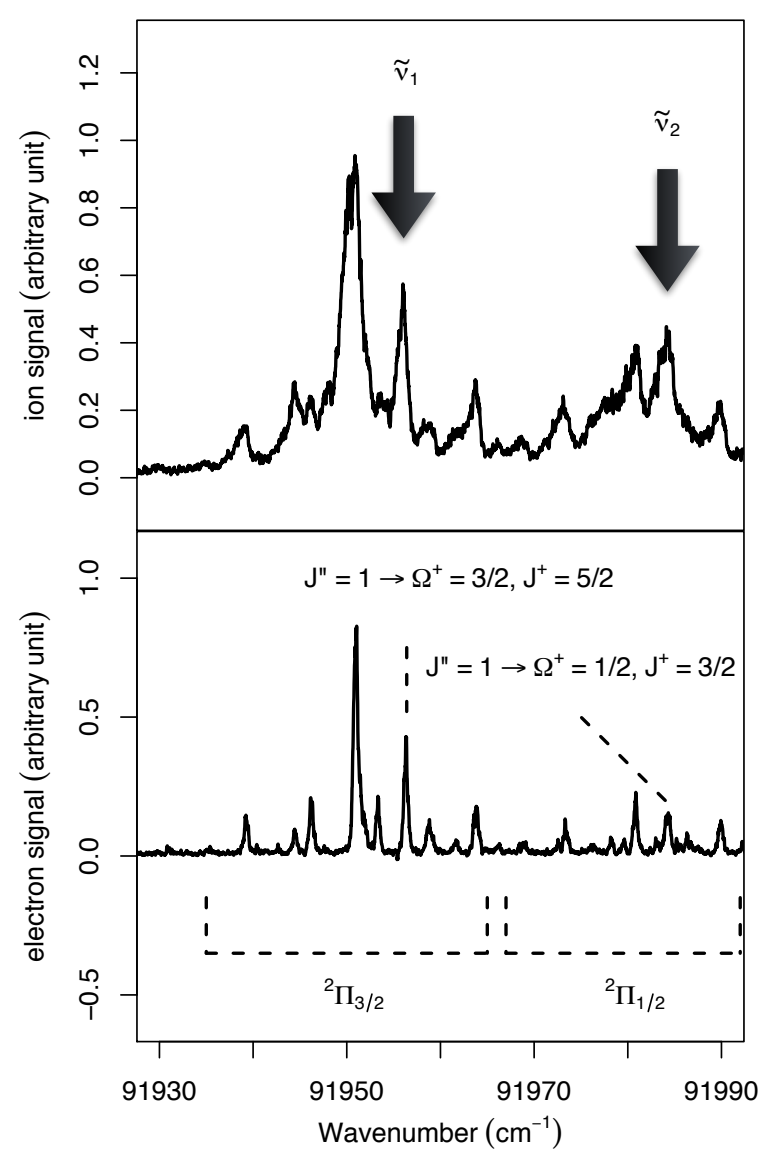

FIG. 3. Comparison of the MATI spectrum (upper trace) and PFI-ZEKE photoelectron spectrum (lower trace, from Ref. [27]) of the $\mathrm{X}^{+}{ }^{2} \Pi_{\mathrm{u}}\left(v^{+}=0\right) \leftarrow \mathrm{X}^{1} \Sigma_{\mathrm{g}}(v=0)$ ionizing transition of $\mathrm{C}_{2} \mathrm{H}_{2}$. Both spectra have been corrected for the field-induced shift of the ionization thresholds.

178 Figure 3 compares the MATI spectrum of the $\mathrm{X}^{+}{ }^{2} \Pi_{\mathrm{u}}$ ${ }_{179}\left(v^{+}=0\right) \leftarrow \mathrm{X}^{1} \Sigma_{\mathrm{g}}(v=0)$ ionizing transition of $\mathrm{C}_{2} \mathrm{H}_{2}$ (up180 per trace) with the high-resolution PFI-ZEKE photoelec181 tron spectrum of the same transition reported by Rup182 per et $a l .^{27}$. Several lines of the MATI spectrum corre183 spond to single transitions, such as the $\mathrm{X}^{+}{ }^{2} \Pi_{3 / 2}\left(J^{+}\right.$ $\left.{ }_{184}=5 / 2\right) \leftarrow \mathrm{X}^{1} \Sigma_{\mathrm{g}}\left(J^{\prime \prime}=1\right)$ transition at the position ${ }_{185} \tilde{\nu}_{1}=91954 \mathrm{~cm}^{-1}$ (arrow marked $\tilde{\nu}_{1}$ in Fig. 3). At this 


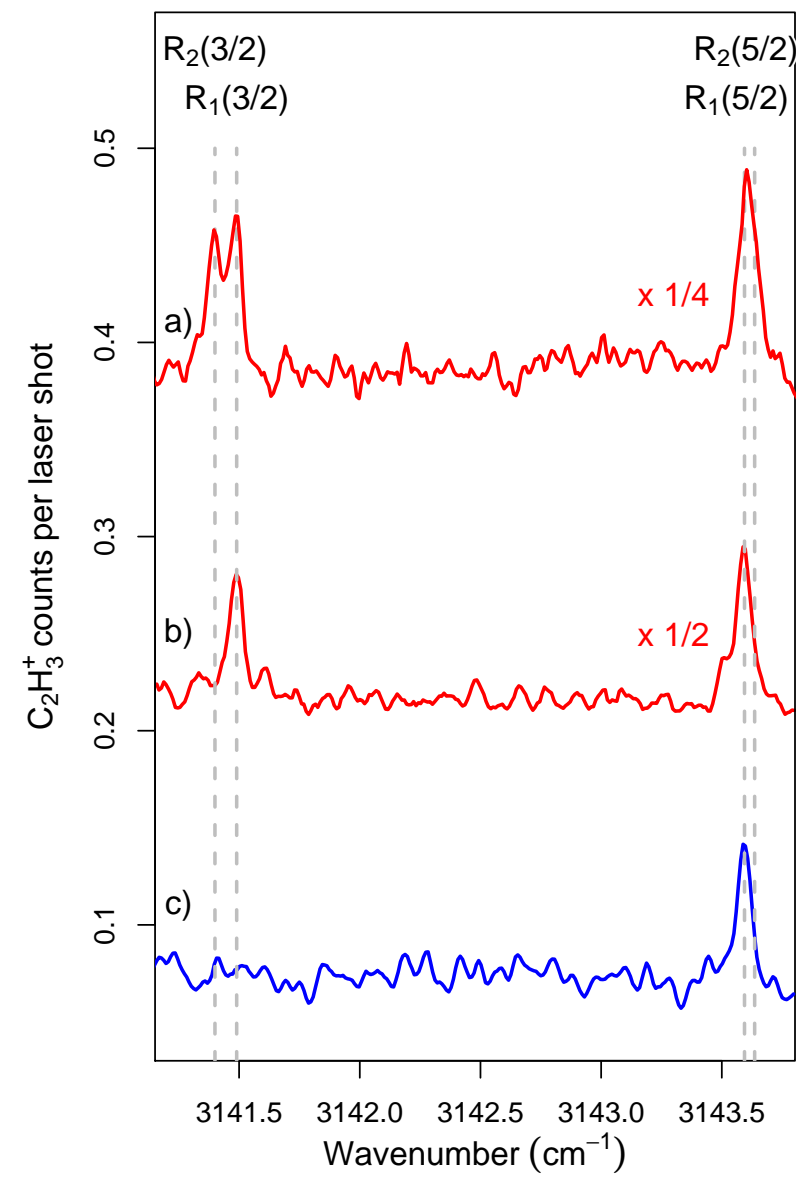

FIG. 4. IR spectra of the fundamental band of the asymmetric C-H stretching mode of $\mathrm{C}_{2} \mathrm{H}_{2}^{+}$. The $\mathrm{C}_{2} \mathrm{H}_{2}^{+}$ions were prepared by photoionization above the ${ }^{2} \Pi_{1 / 2}$ component (a), by photoionization at the position marked $\tilde{\nu}_{1}$ in Fig. 3 (b), and following selection of the $\mathrm{X}^{+2} \Pi_{3 / 2}\left(v^{+}=0, J^{+}=5 / 2\right)$ ionic level by MATI spectroscopy (c). Traces a), b), and c) were obtained by averaging over 1200, 2000, and 1200 laser excitation cycles, respectively.

186 spectral position, the pulse sequence used to form the ion 187 beam guarantees that the ions in the beam are in the $\mathrm{X}^{+}$ ${ }_{188}{ }^{2} \Pi_{3 / 2}\left(J^{+}=5 / 2\right)$ level prior to IR excitation. At the 189 position $\tilde{\nu}_{2}=91982 \mathrm{~cm}^{-1}$ (arrow marked $\tilde{\nu}_{2}$ in Fig. 3), 190 the $\mathrm{C}_{2} \mathrm{H}_{2}^{+}$ions are produced in the $J^{+}=3 / 2$ rotational 191 level of the ${ }^{2} \Pi_{1 / 2}$ spin-orbit component. By turning off 192 the discrimination pulse, one can also generate an ion 193 beam to which all ions contribute that can be generated 194 by direct photoionization at the selected wavenumber.

195 These advantages are illustrated in Fig. 4. Trace a) was 196 measured without discrimination pulse with the VUV 197 wavenumber set at $92080 \mathrm{~cm}^{-1}$, i.e., well above the $\mathrm{X}^{+}$ ${ }_{198}^{2} \Pi_{1 / 2} \leftarrow \mathrm{X}^{1} \Sigma_{\mathrm{g}}$ band. At this position all optically ac-
${ }_{199}$ cessible rotational levels of the $\mathrm{X}^{+}{ }^{2} \Pi_{3 / 2}$ and ${ }^{2} \Pi_{1 / 2}$ spin200 orbit components are generated. The spectral region dis201 played in this spectrum consists of $\mathrm{R}$ transitions from the ${ }_{202} J^{+}=3 / 2$ and $5 / 2$ levels of both spin-orbit components. ${ }_{203}$ When the VUV laser wavenumber is set at $91954 \mathrm{~cm}^{-1}$, 204 i.e., below the onset of the $\mathrm{X}^{+2} \Pi_{1 / 2} \leftarrow \mathrm{X}^{1} \Sigma_{\mathrm{g}}$ band in the 205 MATI spectrum (see Fig. 3), but still without discrimi206 nation pulse, the two $\mathrm{R}_{2}$ lines disappear as can be seen in 207 the middle trace (trace b)) in Fig. 4. The $\mathrm{R}_{1}(5 / 2)$ line is 208 the only one remaining when the VUV laser is tuned to 209 the position of $\tilde{\nu}_{1}$ in Fig. 3 and the discrimination pulse is 210 switched on (trace c) in Fig. 4). This trace thus demon211 strates full rotational state selectivity in the $\mathrm{X}^{+}{ }^{2} \Pi_{3 / 2}$ 212 spin-orbit component.

${ }_{213}$ Full rotational state selectivity can also be achieved in 214 the upper $\left({ }^{2} \Pi_{1 / 2}\right)$ spin-orbit component, as illustrated 215 in Fig. 5. This figure compares the IR spectrum near ${ }_{216}$ the $\mathrm{R}_{1,2}(3 / 2)$ line pairs recorded with the VUV laser 217 wavenumber fixed at the position marked $\tilde{\nu}_{2}$ in Fig. 3 218 with (lower trace) and without (upper trace) discrimi${ }_{219}$ nation pulse. This state selectivity is possible because 220 the $\mathrm{H}_{2}$ gas density in the reaction zone is low enough to 221 ensure single-collision conditions and thus to avoid state 222 redistribution by inelastic collisions prior to the reaction. ${ }_{223}$ The lines observed in the IR spectrum presented in ${ }_{224}$ Figs. 4 and 5 have a full width at half maximum of ${ }_{225} 0.035 \mathrm{~cm}^{-1}$, limited by the bandwidth of the IR laser. 226 In IR spectra recorded by applying the IR laser pulse be${ }_{227}$ fore the field ionization pulse, we did not observe any fre${ }_{228}$ quency shift of the IR transitions but a slight broadening, 229 which we attribute to the presence of the high- $n$ Rydberg 230 electron. The transition wavenumbers, corrected for the 231 estimated Doppler shift of $-0.006 \mathrm{~cm}^{-1}$, are compared 232 in Table I to the more precise transition wavenumbers 233 reported in Ref. [26] with which they are in excellent 234 agreement.

\begin{tabular}{lcc} 
& This work & Ref. [26] \\
\hline $\mathrm{R}_{1}(3 / 2)$ & $3141.488(20)$ & 3141.491 \\
$\mathrm{R}_{2}(3 / 2)$ & $3141.400(20)$ & 3141.402 \\
$\mathrm{R}_{1}(5 / 2)$ & $3143.597(20)$ & 3143.594 \\
$\mathrm{R}_{2}(5 / 2)$ & $3143.629(30)$ & 3143.636 \\
\hline
\end{tabular}

TABLE I. Wavenumbers (in $\mathrm{cm}^{-1}$ ) of the $\mathrm{R}_{1,2}(3 / 2)$ and $\mathrm{R}_{1,2}(5 / 2)$ transitions of the fundamental band of the asymmetric-stretching mode of $\mathrm{C}_{2} \mathrm{H}_{2}^{+}\left(\mathrm{X}^{+}{ }^{2} \Pi_{\mathrm{u}}\right)$.

\section{${ }_{235}$ IV. CONCLUSION}

236 We developed a method and built a new apparatus 237 to record infrared spectra of mass- and state-selected 238 cations. The method relies on the preparation of the 239 individual vibrational and rotational state of molecular 240 cations by photoionization and field-ionization methods 241 and on the detection of the product of reactions induced 242 by the absorption of infrared radiation. The state selec243 tivity of the method reduces spectral congestion, which 


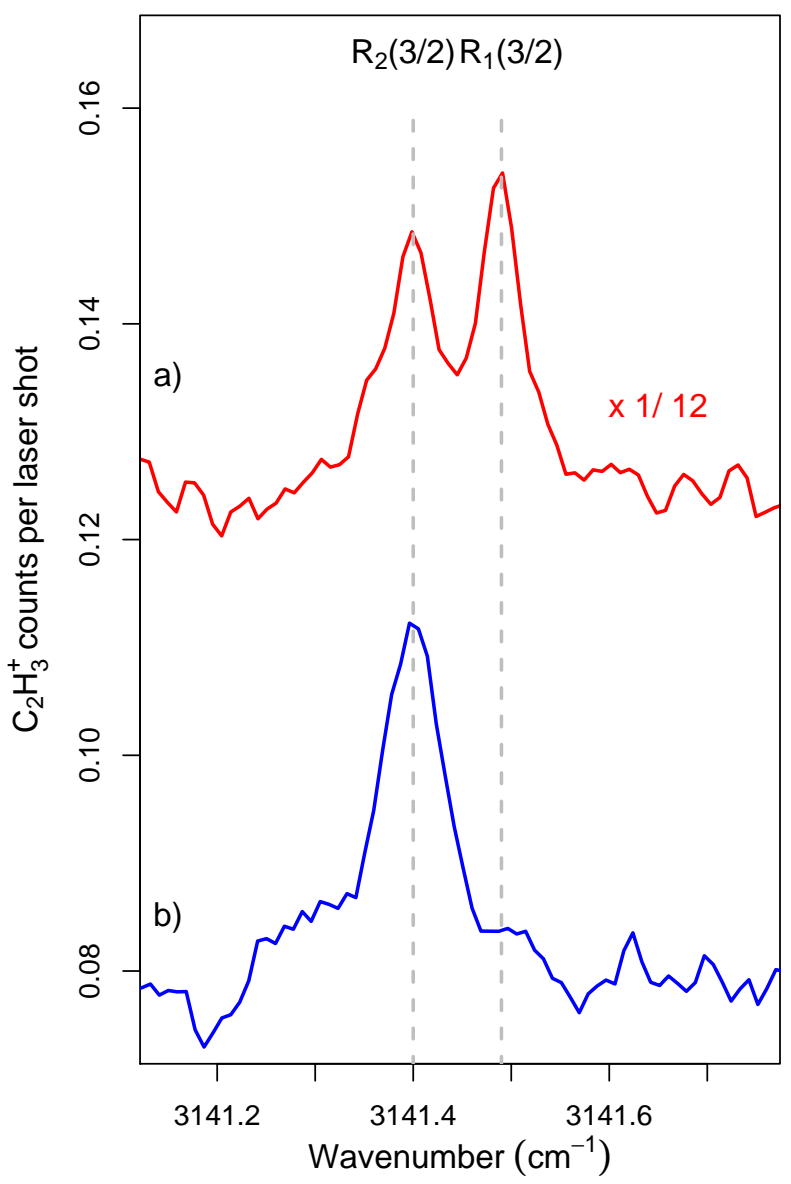

FIG. 5. IR spectra of the fundamental band of the asymmetric C-H stretching mode of $\mathrm{C}_{2} \mathrm{H}_{2}^{+}$. The $\mathrm{C}_{2} \mathrm{H}_{2}^{+}$ions were prepared by photoionization above the ${ }^{2} \Pi_{1 / 2}$ component (a) and following selection of the $\mathrm{X}^{+2} \Pi_{1 / 2}\left(v^{+}=0, J^{+}=3 / 2\right)$ ionic level by MATI spectroscopy (b) at the position marked $\tilde{\nu}_{2}$ in Fig. 3.

244 facilitates assignments. The main obstacle to the general 245 use of this method is the necessity to identify a reac246 tion with the appropriate kinetics and thermodynamical 247 properties.
248 ACKNOWLEDGMENTS

${ }_{249}$ This work is supported financially by the Swiss Na250 tional Science Foundation under project Nr. 200020251159848 .

$252{ }^{1}$ A. Carrington, J. Buttenshaw and R. Kennedy, Mol. Phys. 45, 253753 (1982).

$254 \quad 2$ E. T. White, J. Tang and T. Oka, Science 284, 135 (1999).

$255 \quad{ }^{3}$ R. Signorell and F. Merkt, J. Chem. Phys. 110, 2309 (1999).

${ }_{256}{ }^{4}$ H. J. Wörner and F. Merkt, Angew. Chem. 48, 6404 (2009).

${ }_{257}^{5}$ G. Herzberg, Q. Rev. Chem. Soc. 25, 201 (1971).

${ }_{258}{ }^{6}$ R. J. Saykally, and R. C. Woods, Ann. Rev. Phys. Chem. 32, 259403 (1981).

$260 \quad{ }^{7}$ E. Hirota, Chem. Rev 92, 141 (1992).

${ }_{261}^{8}$ E. Hirota, Annu. Rep. Prog. Chem., Sect. C: Phys. Chem. 96, $26295(2000)$

${ }_{263}{ }^{9} \mathrm{~T}$. Oka, "High-resolution Photoelectron Spectroscopy" in "The 264 Encyclopedia of Mass Spectrometry", Elsevier, Amsterdam, 1, 265217 (2003).

${ }_{266}{ }^{10}$ S. K. Stephenson and R. J. Saykally , Chem. Rev. 105, 3220 267 (2005).

${ }_{268}^{11}$ J. N. Hodges, A. J. Perry, P. A. Jenkins II, B. M. Siller, and B. 269 J. McCall, J. Chem. Phys. 139, 164201 (2013).

${ }_{270}{ }^{12}$ K. Müller-Dethlefs, and E. W. Schlag, Ann. Rev. Phys. Chem. $271 \quad 42,109$ (1991).

${ }_{272}{ }^{13}$ F. Merkt, S. Willitsch and, U. Hollenstein, "High-resolution Pho273 toelectron Spectroscopy" in "Handbook of High-Resolution Spec274 troscopy", Martin Quack (ed.) and Frédéric Merkt (ed.), John 275 Wiley \& Sons, Chichester 3, 1617 (2011).

${ }_{276}{ }^{14}$ A. Dzhonson, D. Gerlich, E. J. Bieske, J. P. Maier, J. Mol. Struct. 277 795, 93 (2006).

${ }_{278}{ }^{15}$ T. R. Rizzo, O. V. Boyarkin, Top. Curr. Chem., 364, 43 (2015). ${ }_{279}{ }^{16}$ S. Schlemmer, T. Kuhn, E. Lescop, D. Gerlich, Int. J. Mass. 280 Spec. 185, 589 (1999).

${ }_{281}{ }^{17} \mathrm{~S}$. Schlemmer, E. Lescop, J. von Richthofen, D. Gerlich, M. A. 282 Smith, J. Chem. Phys. 117, 2068 (2002).

${ }_{283}{ }^{18}$ M. A. Duncan, Int. Rev. Phys. Chem. 22, 407 (2003).

${ }_{284}{ }^{19}$ S. Chakrabarty, M. Holz, E. K. Campbell, A. Banerjee, D. Ger285 lich, and J. P. Maier, J. Phys. Chem. Lett., 4, 4051 (2013).

${ }_{286}^{20}{ }^{20}$. Tong, D. Wild, and S. Willitsch, Phys. Rev. A 22, 023415 (2011).

${ }^{288}{ }^{21}$ A. M. Ricks, G. E. Douberly, P. v. R. Schleyer, and M. A. Duncan, J. Chem. Phys. 132, 051101 (2010).

${ }_{290}^{22}$ D. Gerlich, J. Chem. Soc., Faraday Trans. 89, 2199 (1993).

${ }_{291}{ }^{23}$ S. Willitsch, Int. Rev. Phys. Chem. 31, 175 (2012).

${ }_{292}{ }^{24} \mathrm{~S}$. Willitsch, "Experimental Methods in Cation Spectroscopy" 293 in "Handbook of High-Resolution Spectroscopy", Martin Quack 294 (ed.) and Frédéric Merkt (ed.), John Wiley \& Sons, Chichester 295 3, 1691 (2011).

${ }_{296}{ }^{25} \mathrm{~T}$. Amano, "High-resolution Microwave and Infrared Spec297 troscopy of Molecular Cations " in "Handbook of High-Resolution 298 Spectroscopy", Martin Quack (ed.) and Frédéric Merkt (ed.), 299 John Wiley \& Sons, Chichester 2, 1267 (2011).

${ }_{300}{ }^{26}$ M.-F. Jagod, M. Rösslein, C. M. Gabrys, B. D. Rehfuss, F. Scap301 pini, M. W. Crofton and T. Oka, J. Chem. Phys. 97, 7111 (1992).

${ }_{302}{ }^{27}$ P. Rupper and F. Merkt, Rev. Sci. Instr. 75, 613 (2004).

${ }_{303}{ }^{28}$ L. Zhu and P. Johnson, J. Chem. Phys. 94, 5769 (1991).

${ }_{304}{ }^{29}$ F. Merkt, S. R. Mackenzie, and T. P. Softley, J. Chem. Phys. 99, 3054213 (1993). 